To appear in the Astrophysical Journal

\title{
The Energetics of a Flaring Solar Active Region, and Observed Flare Statistics
}

\author{
M.S. Wheatland \\ School of Physics, University of Sydney, NSW 2006, Australia
}

\begin{abstract}
A stochastic model for the energy of a flaring solar active region is presented, generalising and extending the approach of Wheatland \& Glukhov (1998). The probability distribution for the free energy of an active region is described by the solution to a master equation involving deterministic energy input and random jump transitions downwards in energy (solar flares). It is shown how two observable distributions, the flare frequency-energy distribution and the flare waiting-time distribution, may be derived from the steady-state solution to the master equation, for given choices for the energy input and for the rates of flare transitions. An efficient method of numerical solution of the steady-state master equation is presented. Solutions appropriate for flaring, involving a constant rate of energy input and power-law distributed jump transition rates, are numerically investigated. The flare-like solutions exhibit power-law flare frequency-energy distributions below a high energy rollover, set by the largest energy the active region is likely to have. The solutions also exhibit approximately exponential (i.e. Poisson) waiting-time distributions, despite the rate of flaring depending on the free energy of the system.
\end{abstract}

Subject headings: Sun: flares — Sun: corona — Sun: activity — Methods: statistical

\section{Introduction}

Solar active regions are the sites of occurrence of most solar flares. Large active regions may persist for several solar rotations, and produce dozens of significant flares during a transit of the disk (Richardson 1951). The largest flares are believed to involve the release of more than $10^{27} \mathrm{~J}$ of stored magnetic energy, the energy appearing in accelerated particles, 
heating, bulk motion of material, and radiation (Hudson 1991). The process underlying flares is accepted to be magnetic reconnection, although the details of the process remain the subject of study (Priest \& Forbes 2002).

The dynamical energy balance of solar active regions presents challenges to our understanding. The energy liberated in flares is thought to be excess or 'free' magnetic energy associated with electric current systems in the solar atmosphere, but the origin of the currents, and hence the source of the energy, is not well understood (McClvmont \& Fisher 1987; Tandberg-Hanssen \& Emslie 1988; Leka et al. 1996; Wheatland 2000a; Schrijver et al. 2005). Two popular pictures for energy supply are first that existing coronal magnetic structures are twisted and sheared by photospheric motions, producing currents, and second that new current-carrying magnetic flux emerges through the photosphere. These mechanisms suggest that energy supply to an active region may be described as a continuous process, driven by slow photospheric and sub-photospheric motions. In contrast, flare energy release is rapid and unexpected. An important point is that the size of the downwards jump in energy associated with a flare may be very large, by comparison with the amount of stored active region energy.

The understanding of active region energy balance is hampered by the inability to calculate coronal magnetic energy. In principle the magneto-hydrodynamic (MHD) virial theorem permits the calculation of magnetic energy from vector field values inferred in the chromosphere subject to the assumption that the field is everywhere force free (e.g. Metcalf et al. 2005). However few chromospheric vector field determinations are made, and the reliability of the method is unknown. Methods for modeling coronal magnetic fields from photospheric or chromospheric boundary conditions are being developed (Metcalf et al. 2008; Schrijver et al. 2008), and these may permit estimation of coronal magnetic energy. There has also been recent progress in methods for estimating the rate of supply of energy to an active region directly from observations (e.g. Welsch et al. 2007). For example, in ideal MHD the Poynting flux is $\mathbf{S}=(-\mathbf{u} \times \mathbf{B}) \times \mathbf{B} / \mu_{0}$, where $\mathbf{u}$ is the fluid velocity and $\mathbf{B}$ is the magnetic field, and in principle this quantity may be estimated at the photosphere from observations.

Although we lack detailed quantitative information about the rate of energy supply to, and the energy stored in, active regions, we do have detailed information about solar flare occurrence. The energy released in flares may be estimated (albeit subject to some error), and the rate of occurrence of flares is observed. Two related statistical properties of flares the frequency-energy distribution, and the distribution of times between events - have been studied in some detail, as summarized below.

Studies of the frequency-energy distribution show that it is a power law over many decades in energy (Hudson 1991; Crosby, Aschwanden, \& Dennis 1993; Aschwanden, Dennis, \& Benz 
1998). Specifically the distribution may be written

$$
\mathcal{N}(E)=A E^{-\gamma}
$$

where $\mathcal{N}(E)$ is the number of flares per unit time and per unit energy $E$, the factor $A$ is a (time-dependent) measure of the total flaring rate, and $\gamma \approx 1.5$. Typically this distribution is determined for flares from all active regions on the Sun over some period of time, but it also appears to apply to individual active regions (Wheatland 2000b), which suggests that the power law is intrinsic to the flare mechanism. A popular model explaining the power law is the avalanche model (Lu \& Hamilton 1991; Charbonneau et al. 2001), in which the magnetic field in the corona is assumed to be in a self-organized critical state, and subject to avalanches of small-scale reconnection events.

There has been considerable interest in the flare waiting-time distribution, and more generally in waiting-time distributions for what they reveal about underlying physics in a variety of systems (Sánchez, Newman \& Carreras 2002). Determinations of flare waitingtime distributions have given varied results (Pearce. Rowe \& Yeung 1993; Biesecker 1994; Wheatland. Sturrock \& McTiernan 1998; Boffetta et al. 1999; Moon et al. 2001; Wheatland 2001; Moon et al. 2002). The results suggest that the observed distribution depends on the particular active region and on time, and that it is also influenced by event definition and selection procedures (Wheatland 2001; Buchlin, Galtier, \& Velli 2005; Paczuski, Boettcher, \& Baiesi 2005; Baiesi, Paczuski, \& Stella 2006). For some active regions, the distribution appears to be consistent with a simple Poisson process, i.e. independent events occurring at a constant mean rate (Moon et al. 2001). The corresponding waiting-time distribution is exponential. Other active regions show time-variation in the flaring process, and flare occurrence may be approximated by a piecewise constant, or more generally time-varying Poisson process (Wheatland 2001). On longer time scales a power-law tail is observed for events from the whole Sun (Boffetta et al. 1999). This may be accounted for in terms of a time-dependent Poisson model (Wheatland \& Litvinenko 2002), although some authors have argued that the power law has fundamental significance (Lepreti et al. 2001). We note that the waiting-time distribution has often been considered in isolation from other flare statistics, in particular the frequency-energy distribution.

To understand the statistics of flare occurrence, it is desirable to have a general theory for the energetics of an active region, relating the free energy of the active region to the observed frequency-energy and waiting-time distributions. Rosner \& Vaiana (1978) presented the first model of this kind, which was analogous to Fermi acceleration. In this model active regions experience exponential growth in free energy between flares which occur as a Poisson process in time, depleting all accumulated energy. This gives a power-law flare frequency-energy distribution for large energies, and presupposes Poisson occurrence. Litvi- 
nenko $(1994 ; 1996)$ generalized the model to incorporate different rates of supply of energy to the system, but the Litvinenko models retained the feature that each flare releases all of the free energy. This aspect of the Rosner \& Vaiana (1978) model was criticized by Lu (1995). Wheatland \& Glukhov (1998) introduced a model permitting arbitrary changes in free energy at each flare. The model assumes the free energy $E$ of an active region increases secularly between jump transitions downward in energy (flares), which occur at a rate $\alpha\left(E, E^{\prime}\right)$ for jumps from $E$ to $E^{\prime}$, per unit energy. A master equation describes the steady-state energy balance, and the solution of this equation is the probability distribution $P(E)$ for the free energy. The flare frequency-energy distribution is given by the convolution

$$
\mathcal{N}(E)=\int_{E}^{\infty} P\left(E^{\prime}\right) \alpha\left(E^{\prime}, E^{\prime}-E\right) d E^{\prime} .
$$

Wheatland \& Glukhov (1998) investigated solutions to the master equation for constant energy input and for a choice of transition rates $\alpha\left(E, E^{\prime}\right) \sim\left(E-E^{\prime}\right)^{-\gamma}$, which leads to power-law behavior $\mathcal{N}(E) \sim E^{-\gamma}$ (below a high energy rollover set by the highest energy an active region is likely to have). Wheatland \& Glukhov (1998) did not determine a waiting-time distribution for the model. It was argued that the derived power-law solutions are consistent with an avalanche-type model, and avalanche models have simple Poisson waiting-time statistics (Wheatland, Sturrock \& McTiernan 1998; Sánchez, Newman \& Carreras 2002). However, this presents a puzzle: the total rate of flaring in the Wheatland \& Glukhov (1998) model is given by

$$
\lambda(E)=\int_{0}^{E} \alpha\left(E, E^{\prime}\right) d E^{\prime}
$$

which depends on the energy of the system. Hence it is expected that the occurrence of flares is not strictly Poisson, since the occurrence of a flare changes the energy of the system, and hence the instantaneous total rate of flaring. Non-Poisson waiting-time statistics might then be expected.

Recently Daly \& Porporato (2007) demonstrated how to determine steady-state waitingtime distributions for continuous time processes with arbitrary jump transitions. The Daly \& Porporato (2007) theory is quite general, applying to any system described by a single time-dependent stochastic variable $x(t)$ following a deterministic trajectory interrupted by positive or negative jumps of random timing and size. The probability distribution $P(x, t)$ for $x(t)$ is described by a master equation, and the waiting-time distribution for jumps in the steady state may be obtained from the solution to the steady-state master equation. Daly \& Porporato (2007) demonstrated the theory in application to simple models for human attention, for voltage across a nerve membrane, and for soil moisture content associated with rainfall events. In each case the models were analytic, involving simple solutions to the master equation. In this paper the Daly \& Porporato (2007) theory is applied to the Wheatland \& Glukhov 
(1998) model for active region free energy, and waiting-time distributions are derived for solutions of relevance to flares. The application of the theory is relatively straightforward: the Wheatland \& Glukhov (1998) model is in the class of models considered by Daly \& Porporato (2007), the time-dependent stochastic variable being the active region energy $E(t)$. Minor modifications to the theory are required because $E$ is a positive definite quantity, and because the jump transitions in $E$ are always negative. A specific difficulty in applying the theory is that for the power-law form for rate transitions relevant for flares, the master equation is not amenable to analytic solution. In this paper an efficient numerical method of solution of the steady state master equation is presented and applied. This paper also considers a more

general form for flare transition rates than considered in Wheatland \& Glukhov (1998). The results resolve the puzzle outlined above concerning whether the model produces an exponential waiting-time distribution.

The sections of the paper are as follows. Section 2 presents the model, starting with the time-dependent master equation $(\$ 2.1)$. Section 2.2 shows how steady-state waiting-time distribution may be obtained, $\S 2.3$ presents simple analytic solutions illustrating the theory, and $\S 2.4$ considers the information provided by moments of the master equation. Section 3 presents flare-like solutions to the master equation, starting with a justification of appropriate choices for the rates of transitions and for the energy supply rate ( $\$ 3.1$. Section 3.2 describes the numerical method, and $\S 3.3$ presents the results. Section 4 discusses the results, and their significance for understanding solar flares.

\section{Model}

\subsection{Master equation}

An active region is modeled as a system with free energy $E(t)$ which evolves in time due to secular energy input and jumps downward in energy at random times and of random sizes. The system is described by the time-dependent master equation (Van Kampen 1992; Gardiner 2004) for the probability distribution $P(E, t)$ of the free energy:

$$
\begin{aligned}
\frac{\partial P(E, t)}{\partial t}= & -\frac{\partial}{\partial E}[\beta(E, t) P(E, t)]-\lambda(E) P(E, t) \\
& +\int_{E}^{\infty} P\left(E^{\prime}, t\right) \alpha\left(E^{\prime}, E\right) d E^{\prime},
\end{aligned}
$$

where $\beta(E, t)$ describes the energy input rate at time $t, \alpha\left(E, E^{\prime}\right)$ describes the rate of flare jumps from $E$ to $E^{\prime}$, and $\lambda(E)$ is the total rate of flaring, given by equation (3). The terms on the right hand side of equation (4) describe the system gradually increasing in energy 
due to energy input, falling to a lower energy due to a flare, and falling from a higher energy due to a flare, respectively. This is the time-dependent version of the master equation given in Wheatland \& Glukhov (1998).

Following Daly \& Porporato (2007), we note that the system is also described by the stochastic differential equation

$$
\frac{d E}{d t}=\beta(E, t)-\Lambda(E, t)
$$

where $\Lambda(E, t)=\sum_{i=1}^{N(t)} \Delta E_{i} \delta\left(t-t_{i}\right)$ describes accumulated losses in energy due to flaring, $\delta(x)$ is the delta function, and where the times $t_{i}$ are given by a state-dependent Poisson process with occurrence rate $\lambda[E(t)]$. The jump amplitudes $\Delta E$ are distributed according to the (state-dependent) distribution $h(\Delta E, E)$, defined by

$$
\alpha(E, E-\Delta E)=\lambda(E) h(\Delta E, E)
$$

so that $\int_{0}^{E} h(\Delta E, E) d(\Delta E)=1$.

\subsection{Steady-state waiting-time distribution}

Daly \& Porporato (2007) showed how — assuming a steady state - the waiting-time distribution for the jump transitions may be derived. In this section we briefly re-iterate the theory, as it applies to the present model.

Consider a deterministic trajectory described by equation (5), starting at energy $E_{s}$ and ending at a higher energy $E_{e}$, the instant before a jump occurs. The distribution $p_{e}(E)$ of final energies $E_{e}$ is given by the rate of jumping at a given energy divided by the mean total rate of jumping, i.e.

$$
p_{e}(E)=\frac{\lambda(E) P(E)}{\langle\lambda\rangle},
$$

where $P(E)$ is the steady-state solution to the master equation (4) and

$$
\langle\lambda\rangle=\int_{0}^{\infty} \lambda(E) P(E) d E
$$

is the mean total rate. The distribution $p_{s}(E)$ of starting energies $E_{s}$ is then given by $p_{e}(E)$ together with the distribution of jumps $h(\Delta E, E)$ :

$$
p_{s}(E)=\int_{E}^{\infty} p_{e}\left(E^{\prime}\right) h\left(E^{\prime}-E, E^{\prime}\right) d E^{\prime},
$$


which using equations (6) and (17) may be rewritten as

$$
p_{s}(E)=\frac{1}{\langle\lambda\rangle} \int_{E}^{\infty} P\left(E^{\prime}\right) \alpha\left(E^{\prime}, E\right) d E^{\prime} .
$$

The waiting-time distribution is given by

$$
p_{\tau}(\tau)=-\frac{d \mathcal{F}}{d \tau}
$$

with

$$
\mathcal{F}(\tau)=\int_{0}^{\infty} p_{\lambda}(E, \tau) d E
$$

where $p_{\lambda}(E, t)$ is the solution to

$$
\frac{\partial p_{\lambda}(E, t)}{\partial t}=-\frac{\partial}{\partial E}\left[\beta(E) p_{\lambda}(E, t)\right]-\lambda(E) p_{\lambda}(E, t)
$$

with the initial condition $p_{\lambda}(E, 0)=p_{s}(E)$. Equation (13) describes the evolution of the system before a flaring jump occurs, i.e. over the deterministic trajectory starting at energy $E_{s}$ and ending at energy $E_{e}$.

A simpler form for the waiting-time distribution may be obtained when $\beta(E)=\beta_{0}$, a constant. Solution of equation (13) by characteristics then gives

$$
p_{\lambda}(E, t)=p_{s}\left(E-\beta_{0} t\right) \exp \left\{-\int_{0}^{t} \lambda\left[E-\beta_{0}(t-s)\right] d s\right\},
$$

assuming $E \geq \beta_{0} t$, and $p_{\lambda}(E, t)=0$ otherwise. In this case

$$
\mathcal{F}(\tau)=\int_{0}^{\infty} p_{s}(u) f(u, \tau) d u
$$

where

$$
f(u, \tau)=\exp \left[-\int_{0}^{\tau} \lambda\left(\beta_{0} s+u\right) d s\right]
$$

so the waiting-time distribution is

$$
p_{\tau}(\tau)=\int_{0}^{\infty} p_{s}(u) \lambda\left(\beta_{0} \tau+u\right) f(u, \tau) d u .
$$

\subsection{Steady-state analytic solutions}

Two analytic examples illustrate the application of the theory. The examples are not relevant for flares, because they do not produce power-law frequency-energy distributions, but they show how Poisson and non-Poisson waiting-time distributions may be obtained. 
The case $\beta(E)=\beta_{0}$ and $\alpha\left(E, E^{\prime}\right)=\alpha_{0}$ (where $\alpha_{0}$ and $\beta_{0}$ are constants) was considered by Wheatland \& Glukhov (1998). In this case equation (3) gives $\lambda(E)=\alpha_{0} E$, so the total rate of jumps is energy dependent and the waiting-time distribution will not correspond to a simple Poisson process. The analytic solution to the steady-state master equation is

$$
P(E)=a E e^{-\frac{1}{2} a E^{2}},
$$

with $a=\alpha_{0} / \beta_{0}$, and from equation (2) the frequency-energy distribution for jumps is a Gaussian:

$$
\mathcal{N}(E)=\alpha_{0} e^{-\frac{1}{2} a E^{2}} .
$$

From equation (8) the mean total rate is $\langle\lambda\rangle=\left(\alpha_{0} \beta_{0}\right)^{1 / 2}$, and using equations (77) and (10) the distributions of end- and start-energies for deterministic trajectories are

$$
p_{e}(E)=\left(\frac{2}{\pi}\right)^{1 / 2} a^{3 / 2} E^{2} e^{-\frac{1}{2} a E^{2}}
$$

and

$$
p_{s}(E)=\left(\frac{2}{\pi}\right)^{1 / 2} a^{1 / 2} e^{-\frac{1}{2} a E^{2}}
$$

respectively. Using equations (17) and (21) it follows that the waiting-time distribution is also a Gaussian:

$$
p_{\tau}(\tau)=\left(\frac{2 \alpha_{0} \beta_{0}}{\pi}\right)^{1 / 2} e^{-\frac{1}{2} \alpha_{0} \beta_{0} \tau^{2}}
$$

As a second example, we consider the case $\beta(E)=\beta_{0}$ and $\lambda(E)=\lambda_{0}$ (where $\beta_{0}$ and $\lambda_{0}$ are constants). Since the total rate is constant the waiting-time distribution must be

$$
p_{\tau}(\tau)=\lambda_{0} e^{-\lambda_{0} \tau},
$$

i.e. jumps occur in time as a simple Poisson process. From equation (3) this case requires $\alpha\left(E, E^{\prime}\right)=\lambda_{0} / E$. The corresponding solution to the steady-state master equation is

$$
P(E)=b^{2} E e^{-b E},
$$

with $b=\lambda_{0} / \beta_{0}$, and from equation (2) the frequency-energy distribution of jumps is exponential:

$$
\mathcal{N}(E)=b \lambda_{0} e^{-b E} .
$$

The mean total rate of jumps given by equation (8) is $\langle\lambda\rangle=\lambda_{0}$, and using equations (7) and (10) we have $p_{e}(E)=b^{2} E e^{-b E}$ and $p_{s}(E)=b e^{-b E}$. From equation (17) it follows that the waiting-time distribution is indeed given by equation (23). 


\subsection{Moments of the master equation}

Moments of the master equation give useful information about the global behavior of solutions (Wheatland \& Litvinenko 2001).

The zeroth moment, obtained by integrating equation (44) over all energies, gives the trivial result

$$
\frac{d}{d t} \int_{0}^{\infty} P(E, t) d E=0,
$$

i.e. normalization is preserved, provided $\beta(E, t) P(E, t)$ goes to zero as $E \rightarrow 0$ and $E \rightarrow \infty$.

The first moment, obtained by multiplying equation (4) by $E$ and integrating over all energies, gives the simple statement of global energy balance:

$$
\frac{d}{d t}\langle E\rangle=\langle\beta\rangle-\langle r\rangle
$$

where for any quantity $f=f(E, t)$, the mean $\langle f\rangle$ is defined by

$$
\langle f\rangle=\int_{0}^{\infty} f(E, t) P(E, t) d E,
$$

and where

$$
r(E)=\int_{0}^{E}\left(E-E^{\prime}\right) \alpha\left(E, E^{\prime}\right) d E^{\prime}
$$

is the total rate of energy loss at energy $E$. Equation (27) requires $\beta(E, t) P(E, t)$ to go to zero as $E \rightarrow 0$ and $E \rightarrow \infty$. In the steady state equation (27) gives

$$
\langle\beta\rangle=\langle r\rangle \text {. }
$$

\section{Flare-like solutions}

In the following we consider solutions to the steady-state master equation which may be of relevance to solar flares.

\subsection{Choices appropriate for flares}

We restrict attention to the case $\beta(E)=\beta_{0}$, a constant. The motivation is that active regions are externally driven, i.e. the energy is supplied from the sub-photosphere by external processes. In the absence of a back reaction, it is then expected that the energy supply rate 
does not depend on the state of the system. In passing we note that in general the energy supply rate may depend on time. However, in this section we consider only steady-state solutions to the master equation. We return to this point in $\S 4$.

We consider the form

$$
\alpha\left(E, E^{\prime}\right)=\alpha_{0} E^{\delta}\left(E-E^{\prime}\right)^{-\gamma} \theta\left(E-E^{\prime}-E_{c}\right)
$$

for the flare transition rate, where $E_{c}$ is a low-energy cutoff, and $\theta(x)$ is the step function. The case $\delta=0$ was considered in Wheatland \& Glukhov (1998). The motivation for equation (31) is that it may describe an avalanche-type system, in which energy transitions are intrinsically power-law distributed. The power law is assumed to originate in the microphysics of the flare process, and must be assumed at the level of this model. [This is in contrast to models which attempt to account for the power law, e.g. Rosner \& Vaiana (1978).] The low-energy cutoff $E_{c}$ is needed to ensure $\lambda(E)$ is finite. The $E^{\delta}$ factor represents a possible dependence of the transition rate on the energy of the system. It is plausible that an avalanche-type system with more energy is more likely to contain unstable sites, and hence will flare at a higher rate. In the following we take $\gamma=1.5$ in every instance, and consider two cases: $\delta=0$, following Wheatland \& Glukhov (1998); and $\delta=1$. The choice of transition rates (31) leads to the total flaring rate

$$
\lambda(E)=\frac{\alpha_{0}}{\gamma-1} E^{\delta}\left(E_{c}^{-\gamma+1}-E^{-\gamma+1}\right) .
$$

Hence the rate of occurrence of flares is energy-dependent, and the waiting-time distribution will not correspond to a simple Poisson process, as pointed out in $\S 1$.

Application of the first moment with the choice of transition rates (31) leads to a simple estimate for the mean energy of the system (Wheatland \& Litvinenko 2002). Specifically, from equation (29) we have

$$
r(E) \approx \frac{\alpha_{0}}{2-\gamma} E^{\delta+2-\gamma}
$$

for $E \gg E_{c}$. Taking averages and making the approximation $\left\langle E^{\delta+2-\gamma}\right\rangle \approx\langle E\rangle^{\delta+2-\gamma}$ together with $\langle\beta\rangle=\beta_{0}$ in equation (30) gives

$$
\langle E\rangle \approx\left(\frac{2-\gamma}{\alpha_{0} / \beta_{0}}\right)^{\frac{1}{\delta+2-\gamma}}
$$

Substituting equation (31) into equation (2) leads to the form for the flare frequencyenergy distribution

$$
\mathcal{N}(E)=\alpha_{0} E^{-\gamma} \int_{E}^{\infty}\left(E^{\prime}\right)^{\delta} P\left(E^{\prime}\right) d E^{\prime}
$$


for $E \geq E_{c}$. Hence it follows that the frequency-energy distribution will be a power law with index $\gamma$ up to energies $E$ at which $P(E)$ becomes very small. Equation (34) provides a crude estimate (a lower bound) for the energy at which the frequency-energy distribution is expected to depart from power law behavior.

\subsection{Numerical method}

The steady-state master equation may be non-dimensionalized by introducing new variables $\bar{E}=E / E_{0}, \bar{P}=P E_{0}, \bar{t}=\beta_{0} t / E_{0}$, and $\bar{\alpha}=E_{0}^{2} \alpha / \beta_{0}$, where $E_{0}$ is a chosen scale for

energy. [For the solutions corresponding to equation (31) we take $E_{0}=E_{c}$.] This procedure gives

$$
\frac{d \bar{P}}{d \bar{E}}+\overline{\lambda P}-\int_{\bar{E}}^{\infty} \bar{P}\left(\bar{E}^{\prime}\right) \bar{\alpha}\left(\bar{E}^{\prime}, \bar{E}\right) d \bar{E}^{\prime}=0
$$

where

$$
\bar{\lambda}=\int_{0}^{\bar{E}} \bar{\alpha}\left(\bar{E}, \bar{E}^{\prime}\right) d \bar{E}^{\prime} .
$$

Hereafter we assume non-dimensional equations, but omit the bars.

Equation (36) is linear in $P(E)$, and hence may be solved by discretizing in energy and solving a coupled system of linear equations. These equations must be supplemented by the normalization condition on $P(E)$. Direct back-substitution provides an efficient method of solution, and the details of the procedure are given in Appendix A. In Wheatland \& Glukhov (1998) the steady-state master equation was solved by a relaxation procedure, but the approach given here is more numerically efficient.

The flare frequency-energy distribution is obtained from the solution for $P(E)$ via numerical evaluation of equation (21). The waiting-time distribution is similarly determined via numerical evaluation of equation (17), using an analytic form for $f(u, \tau)$ obtained from equation (16). All numerical integrations use the extended trapezoidal rule. The numerical solution was tested on the analytic cases given in $\S 2.3$.

\subsection{Results}

First we consider the case $\delta=0$, following Wheatland \& Glukhov (1998). Figure 1 illustrates the numerical solution of the steady-state master equation (36) for the case $\alpha_{0}=$ 0.1, which is one of the two cases considered in Wheatland \& Glukhov (1998). The upper panel shows the probability distribution $P(E)$ for active region energy (as a linear-log plot), 
the middle panel shows the flare frequency-energy distribution $\mathcal{N}(E)$ (as a log-log plot), and the lower panel shows the waiting-time distribution $p_{\tau}(\tau)$ (as a log-linear plot). As explained in $\S 3.1$, the frequency-energy distribution is expected to be a power law with index $\gamma=1.5$ below energies at which $P(E)$ becomes small, and the expression (34) provides a lower bound for the departure from power-law behavior. The lower bound is shown in the upper and middle panels by a vertical line. The upper and middle panels confirm the results of Wheatland \& Glukhov (1998). The lower panel shows the waiting-time distribution $p_{\tau}(\tau)$ (solid curve) as well as the Poisson distribution $\langle\lambda\rangle e^{-\langle\lambda\rangle \tau}$ (dotted line) corresponding to the mean rate of flaring implied by the form of $\lambda(E)$ and the solution for $P(E)$ [see equation (8)]. Note that the units for time in the lower panel are $E_{c} / \beta_{0}$, following the nondimensionalization in $\S 3.2$, The waiting-time distribution for the model is approximately Poisson, although there is a slight deficiency of long waiting-times by comparison with the Poisson distribution.

Figure 2 shows the case $\delta=0$ and $\alpha_{0}=0.02$, which is the other case considered by Wheatland \& Glukhov (1998), in the same format as Figure 1. For a lower value of $\alpha$ the system flares less often and hence is more likely to have larger energy. Hence the distribution $P(E)$ shown in the upper panel is shifted to higher energy and has a higher mean. The flare frequency-energy distribution (middle panel) is a power law over more decades in energy than for the case $\alpha_{0}=0.1$. These results are consistent with the findings of Wheatland \& Glukhov (1998). The lower panel shows the waiting-time distribution (solid curve) as well as the Poisson distribution corresponding to $\langle\lambda\rangle$ (dotted), although the two curves are almost indistinguishable.

The results in Figures 11 and 2 suggest that the $\delta=0$ model has a waiting-time distribution which is close to being strictly Poisson (exponential), and that the approximation becomes better for smaller values of $\alpha_{0}$. This may be understood in terms of the expression (32). For $E \gg E_{c}$ the total rate is $\lambda(E) \approx \alpha_{0} E_{c}^{-\gamma+1} /(\gamma-1)$, which is constant, in which case a Poisson waiting-time distribution is expected. For smaller values of $\alpha_{0}$ the system is more likely to have larger energy, and hence the approximation $E \gg E_{c}$ will be better. Figure 3 illustrates this explanation for the case $\delta=0, \alpha_{0}=0.02$. The solid curve shows the total rate as a function of energy, and the dashed line shows the mean total rate $\langle\lambda\rangle$. The energy distribution $P(E)$ is shown, with arbitrary normalization, by the dotted curve. We see that, over the range of energy for which the distribution $P(E)$ is substantial, the total rate is approximately constant and equal to the mean total rate. These results resolve the puzzle identified in $\S[$, the waiting-time distribution for the model is not strictly Poisson, but is a good approximation to an exponential.

Next we consider the case $\delta=1$, to examine what happens when the rate of flare 
transitions increases with the energy of the system. Figures 4 and 5 show the cases $\alpha_{0}=10^{-3}$ and $\alpha_{0}=10^{-5}$ respectively, in the same format as Figures 1 and 2. First consider Figure 4 . The energy distribution $P(E)$ shown in the upper panel is qualitatively similar to the $\delta=0$ case, although the distribution declines more rapidly at higher energies, so that it is more skewed in a linear-log representation. Since the rate of transitions increases with energy, the system is less likely to be found at very large energies, and this explains the rapid decline. The middle panel shows the flare frequency-energy distribution $\mathcal{N}(E)$, which is a power law with index $\gamma$ below a high energy rollover. The estimate (34) for the mean of the distribution (vertical line) provides a lower bound for the departure from power law behavior. The lower panel shows the waiting-time distribution $p_{\tau}(\tau)$, and the Poisson distribution implied by $\langle\lambda\rangle$. The waiting-time distribution is approximately Poisson, but has a deficit of long waiting times. Figure 5 illustrates the case with reduced flare transition rates. The distribution $P(E)$ (upper panel) is shifted to higher energies, and is again quite skewed in the linear-log representation. The frequency-energy distribution $\mathcal{N}(E)$ (middle panel) is a power law over more decades in energy. The waiting-time distribution $p_{\tau}(\tau)$ (lower panel) is again approximately exponential, but departs somewhat from the Poisson model, including showing an excess of long waiting times.

The results in the lower panels of Figures 4 and 5 suggest that, for the $\delta=1$ model, the waiting-time distribution is approximately exponential but shows some departure from the Poisson case depending on the parameters of the model. The approximate Poisson behavior is perhaps surprising because in this case the total rate of flaring [given by equation (32)] varies approximately linearly with $E$ (for $E \gg E_{c}$ ). Figure 6 illustrates this for the case $\delta=1, \alpha_{0}=10^{-5}$, using the same format as Figure 3. The total rate (solid curve) increases substantially over the range of energies the system is likely to have [the dotted curve shows $P(E)$ ], and may be substantially different to $\langle\lambda\rangle$ (the dashed line). However, $p_{\tau}(\tau)$ is defined by a complicated average of the rate over energy, which is different for different waiting times [see equation (17)], and the numerical results show that the resulting waiting-time distribution is approximately Poisson.

\section{Discussion}

A stochastic model is presented for the free magnetic energy of a flaring solar active region. The energy of an active region is assumed to grow deterministically between random flare events at which the energy jumps downwards by an amount equal to the flare energy. Flares jumps occur from energy $E$ to $E^{\prime}$ with a rate $\alpha\left(E, E^{\prime}\right)$ per unit time and per unit energy, and energy input occurs at a rate $\beta(E)$. Active region energy is then described by 
a distribution $P(E)$ which is the steady state solution to a master equation. This distribution determines two observable distributions, namely the flare frequency-energy distribution, and the waiting-time distribution. The model generalizes and extends the approach of Wheatland \& Glukhov (1998). Novel aspects of the work presented here include the determination of waiting-time distributions [following general theory presented by Daly \& Porporato (2007)], consideration of a more general form for the rate of flare transitions, and introduction of an efficient method of numerical solution of the steady-state master equation.

The form $\alpha\left(E, E^{\prime}\right)=\alpha_{0} E^{\delta}\left(E-E^{\prime}\right)^{-1.5}$ for flare transitions is investigated, for the cases $\delta=0$ and $\delta=1$. The case $\delta=0$ was considered by Wheatland \& Glukhov (1998), motivated by the avalanche model. For both cases the model is shown to produce powerlaw flare frequency-energy distributions below a rollover at high energies, due to the active region having a finite energy. For both cases the waiting-time distribution is approximately exponential (Poisson). For the case $\delta=0$ this may be understood in that the total rate $\lambda(E)$ is approximately constant for $E \gg E_{c}$, which becomes a good approximation for small $\alpha_{0}$, when the system is more likely to be found at large energies. This result is consistent with the interpretation of this model as avalanche-like, since avalanche models have simple Poisson statistics. For the case $\delta=1$ the interpretation is more complicated, because the total rate varies approximately linearly with energy. However, the waiting-time distribution is determined by an average of rates over the possible energies of the active region, and numerical evaluation shows that the result is approximately exponential.

The general model introduced in $\S 2.1$ includes time dependence in the driving rate, but we have focused on the steady state throughout this paper. Many active regions exhibit large variations in the rate of flaring during a transit of the disk (Wheatland 2001). This behavior is often linked e.g. with the emergence of new magnetic flux (Romano \& Zuccarello 2007), which suggest that it is a response to an increased rate of driving. Hence we have neglected an important aspect of active region energetics. Time-dependent driving will influence the observed waiting-time distribution. In the simplest case time variation might be represented by a piecewise constant variation in the driving rate. If the system adjusts suitably quickly to changes in driving, the steady state solution applies to each piece. The waiting-time distribution is then a weighted sum over the steady-state distributions applying to each piece (Wheatland \& Litvinenko 2001). Based on the results presented in this paper, the waiting-time distribution for active regions is expected to be approximately exponential provided the rate of driving of the system is constant. If the rate of driving is time varying, the distribution will depart from exponential. A time-dependent model will be investigated in future work.

One shortcoming of the model is that it does not describe energy loss from the system 
by mechanisms other than flaring, for example loss due to flux submergence, or quasi-steady background dissipation. However, a simple generalization of the master equation permits this. Specifically, if the secular energy 'input' is replaced by small jumps in energy (which may be positive or negative), then the energy gains and losses may be represented by FokkerPlanck terms in a generalized master or Chapman-Kolmogorov equation. Specifically, the secular energy increase term $-\partial[\beta(E, t) P(E, t)] / \partial E$ on the right hand side of equation (44) may be replaced by a pair of terms $-\partial\left[\beta_{1}(E, t) P(E, t)\right] / \partial E+\frac{1}{2} \partial^{2}\left[\beta_{2}(E, t) P(E, t)\right] / \partial E^{2}$, where the coefficients $\beta_{i}(E, t), i=1,2$ represent first and second moments of energy changes associated with the small jump transitions (Van Kampen 1992; Gardiner 2004). It is straightforward to solve the resulting generalize master equation by discretization and solution of the resulting linear system, extending the approach presented in the Appendix. However, in this case the Daly \& Porporato (2007) method for determining the steady-state waiting-time distribution needs to be modified. This model will be investigated more completely in future work.

The results presented here show how it is possible to construct a model for active region energetics which directly predicts observable flare statistics, namely the flare frequencyenergy, and waiting-time distributions. In principle the observations may be used to determine the energy supply and flare energy release terms in the model, i.e. $\beta(E, t)$ and $\alpha\left(E, E^{\prime}\right)$. However, the observations are not really precise or unambiguous enough to identify these terms with certainty. In particular, the interpretation of the waiting-time distribution is complicated by the time dependence of the energy supply. The situation would be improved by an ability to estimate coronal free energy and the rate of supply of energy to active regions from observations. Reliable methods for estimating these quantities are the subject of current research (Welsch et al. 2007; Schrijver et al. 2008). If such methods are developed, the theory developed here will be of greater significance. It may provide valuable insight into the flare mechanism, as well as being of practical benefit for flare prediction.

The author thanks Ian Craig for pointing out that the master equation can be solved as a linear system by back-substitution, and a referee for helpful comments which improved the presentation. 


\section{A. Appendix}

\section{Numerical solution of the steady-state master equation}

Discretizing equation (36) at energies $E_{i}=i \Delta$ gives

$$
\frac{P_{i+1}-P_{i-1}}{2 \Delta}+\lambda_{i} P_{i}-\frac{1}{2} \Delta \sum_{j=i}^{N-2}\left(P_{j} \alpha_{j, i}+P_{j+1} \alpha_{j+1, i}\right)=0
$$

where

$$
\lambda_{i}=\frac{1}{2} \Delta \sum_{j=0}^{i-1}\left(\alpha_{i, j}+\alpha_{i, j+1}\right)
$$

and where $P_{i}=P\left(E_{i}\right)$ and $\alpha_{i, j}=\alpha\left(E_{i}, E_{j}\right)$. Centered differencing is used for the derivative, and the extended trapezoidal rule is used for the integrals. Equation (A1) with $i=1,2, \ldots, N-1$ (and the assumption $P_{N}=0$ ) may be supplemented by the normalization condition

$$
\frac{1}{2} \Delta \sum_{i=0}^{N-2}\left(P_{i}+P_{i+1}\right)=1
$$

to give $N$ linear equations in the $N$ unknowns $P_{0}, P_{1}, P_{2}, \ldots, P_{N-1}$.

The resulting linear system may be solved efficiently by back-substitution as follows. Equation (A1) may be re-written

$$
P_{i-1}=P_{i+1}+2 \Delta\left[\lambda_{i} P_{i}-\frac{1}{2} \Delta \sum_{j=i}^{N-2}\left(P_{j} \alpha_{j, i}+P_{j+1} \alpha_{j+1, i}\right)\right],
$$

which expresses $P_{i-1}$ in terms of $P_{i}, P_{i+1}, P_{i+2}, \ldots, P_{N-2}$. Hence if we assume a value for $P_{N-1}$, we can apply equation (A4) to solve for $P_{N-2}$, and then apply it again to solve for $P_{N-3}$, etc. In this way we can determine $P_{N-1}, P_{N-2}, P_{N-3}, \ldots, P_{0}$ up to an unknown normalization factor. The factor is determined by applying equation (A3). Specifically, the solution is given by $P_{i}^{\prime}$ (with $i=0,1,2, \ldots, N-1$ ), where

$$
P_{i}^{\prime}=\frac{P_{i}}{\frac{1}{2} \Delta \sum_{i=0}^{N-2}\left(P_{i}+P_{i+1}\right)}
$$

\section{REFERENCES}

Aschwanden, M.J., Dennis, B.R., \& Benz, A.O. 1998, ApJ, 497, 972 
Baiesi, M., Paczuski, M., \& Stella, A.L. 2006, Phys. Rev. Lett., 96, 051103

Biesecker, D. 1994, Ph.D. thesis, Univ. New Hampshire

Boffetta, G., Carbone, V., Giuliani, P., Veltri, P., and Vulpiani, A. 1999, Phys. Rev. Lett., 83,4662

Buchlin, E., Galtier, S., \& Velli, M. 2005, A\&A, 436, 355

Charbonneau, P., McIntosh, S. W., Liu, H.-L., \& Bogdan, T. J. 2001, Sol. Phys., 203, 321

Crosby, N.B., Aschwanden, M.J., \& Dennis, B.R. 1993, Sol. Phys., 143, 275

Daly, E., \& Porporato, A. 2007, Phys. Rev. E, 75, 011119

Gardiner, C.W. 2004, Handbook of Stochastic Methods, 3rd ed. (Springer: Berlin)

Hudson, H.S. 1991, Sol. Phys., 133, 357

Leka, K.D., Canfield, R.C., McClymont, A.N., \& van Driel-Gesztelyi, L. 1996, ApJ, 462, 547

Lepreti, F., Carbone, P. \& Veltri, P. 2001, ApJ, 555, L133

Litvinenko, Y.E. 1994, Sol. Phys., 151, 195

Litvinenko, Y.E. 1996, Sol. Phys., 167, 321

Lu, E.T. 1995, ApJ, 447, 416

Lu, E.T. \& Hamilton, R.J. 1991, ApJ, 380, L89

McClymont, A.N., \& Fisher, G.H. 1989, in Yosemite Conference on Outstanding Problems in Solar System Plasma Physics: Theory and Instrumentation, ed. J. Waite, J. Burch, \& R. Moore, Geophys. Monogr. 54 (AGU: Washington, DC)

Metcalf, T.R., Leka, K.D., \& Mickey, D.L. 2005, ApJ, 623, L53

Metcalf, T.R., DeRosa, M.L., Schrijver, C.J., Barnes, G., van Ballegooijen, A.A., Wiegelmann, T., Wheatland, M.S., Valori, G., \& McTiernan, J.M. 2008, Sol. Phys., 247, 269

Moon, Y.-J., Choe, G.S., Yun, H.S., \& Park, Y.D. 2001, JGR Space Phys., 106, 29951

Moon, Y,-J., Choe, G.S., PArk, Y.D., Wang, H., Gallagher, P.T., Chae, J., Yun, H.S., \& Goode, P.R. 2002, ApJ, 574, 434 
Paczuski, M., Boettcher, S., \& Baiesi, M. 2005, Phys. Rev. Lett., 95, 181102

Pearce, G., Rowe, A., \& Yeung, J. 1993, A\&A, 228, 513

Priest, E.R., \& Forbes, T.G. 2002, Astron. Astrophys. Rev., 10, 313

Richardson, R.S. 1951, ApJ, 114, 356

Romano, P. \& Zuccarello, F. 2007, A\&A, 474, 633

Rosner, R., Vaiana, G. 1978, ApJ, 222, 1104

Sánchez, R., Newman, D.E., \& Carreras, B.A. 2002, Phys. Rev. Lett., 88, 068302

Schrijver, C.J., DeRosa, M.L., Title, A.M., \& Metcalf, T.R. 2005, ApJ, 628, 501

Schrijver, C.J., DeRosa, M.L., Metcalf, T., Barnes, G., Lites, B., Tarbell, B., McTiernan, J., Valori, G., Wiegelmann, T., Wheatland, M.S., Amari, T., Aulanier, G., Demoulin, P., Fuhrmann, M., Kusano, K., Regnier, S., \& Thalmann, J.K. 2008, to appear in ApJ

Tandberg-Hanssen, E., \& Emslie, A.G. 1988, The Physics of Solar Flares (Cambridge University Press: Cambridge)

Van Kampen, N.G. 1992, Stochastic Processes in Physics and Chemistry (Elsevier: Amsterdam)

Welsch, B.T., Abbett, W.P., DeRosa, M.L., Fisher, G.H., Georgoulis, M.K., Kusano, K., Longcope, D.W., Ravindra, B., and Schuck, P.W. 2007, ApJ, 670, 1434

Wheatland, M.S., \& Glukhov, S. 1998, ApJ, 494, 858

Wheatland, M.S., \& Litvinenko, Y.E. 2001, ApJ, 550, 109

Wheatland, M.S., Sturrock, P.A., \& McTiernan, J.M. 1998, ApJ, 509, 448

Wheatland, M.S. 2000a, ApJ, 532, 616.

Wheatland, M.S. 2000b, ApJ, 532, 1209.

Wheatland, M.S. 2001, Sol. Phys., 203, 87

Wheatland, M.S., \& Litvinenko, Y.E. 2002, Sol. Phys., 255 

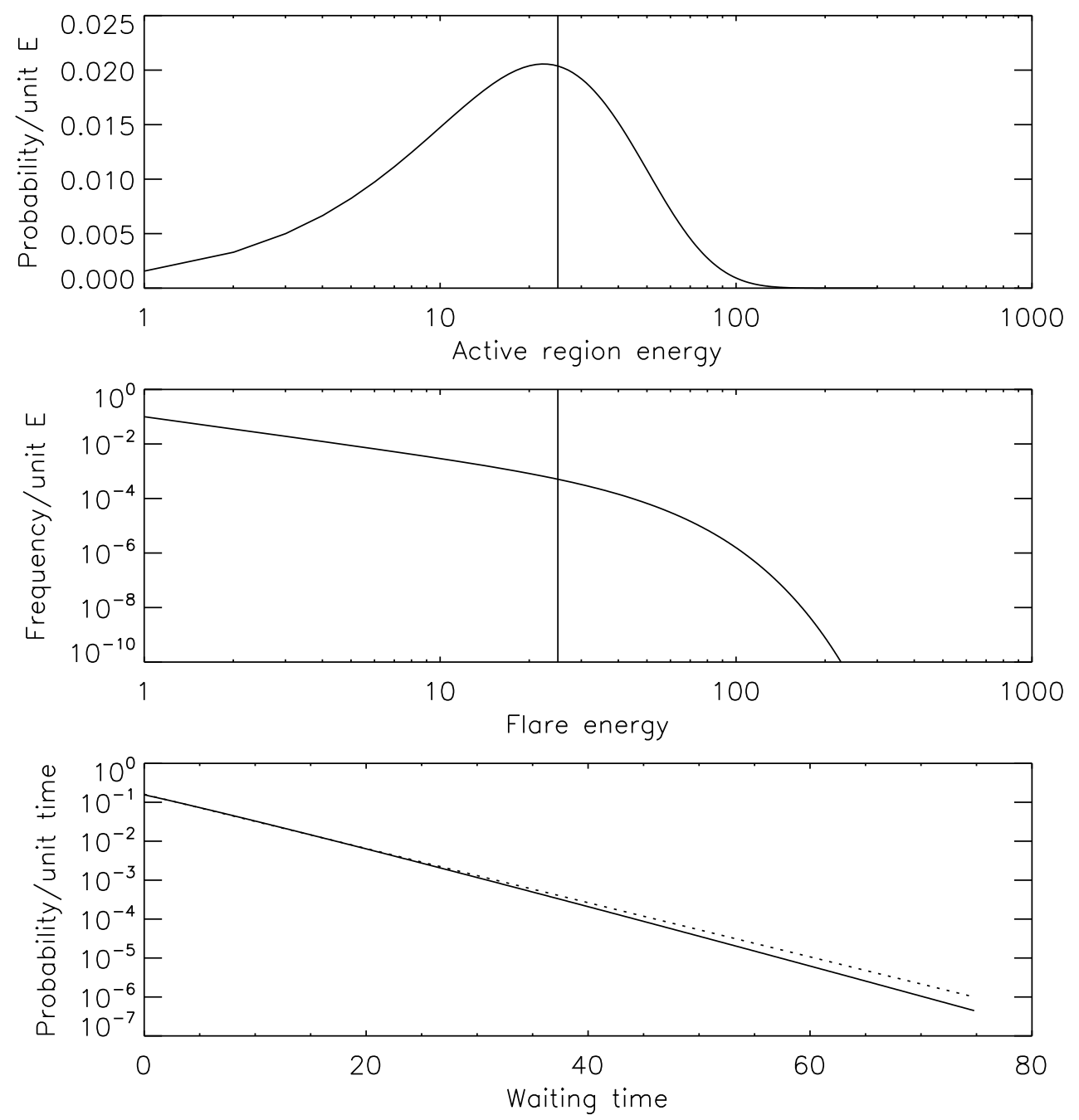

Fig. 1. - Numerical solution to the steady-state master equation for the case $\delta=0, \gamma=$ 1.5, and $\alpha_{0}=0.1$, one of the cases considered in Wheatland \& Glukhov (1998). Upper panel: probability distribution for free energy $P(E)$; middle panel: flare frequency-energy distribution $\mathcal{N}(E)$; lower panel: flare waiting-time distribution $p_{\tau}(\tau)$. 

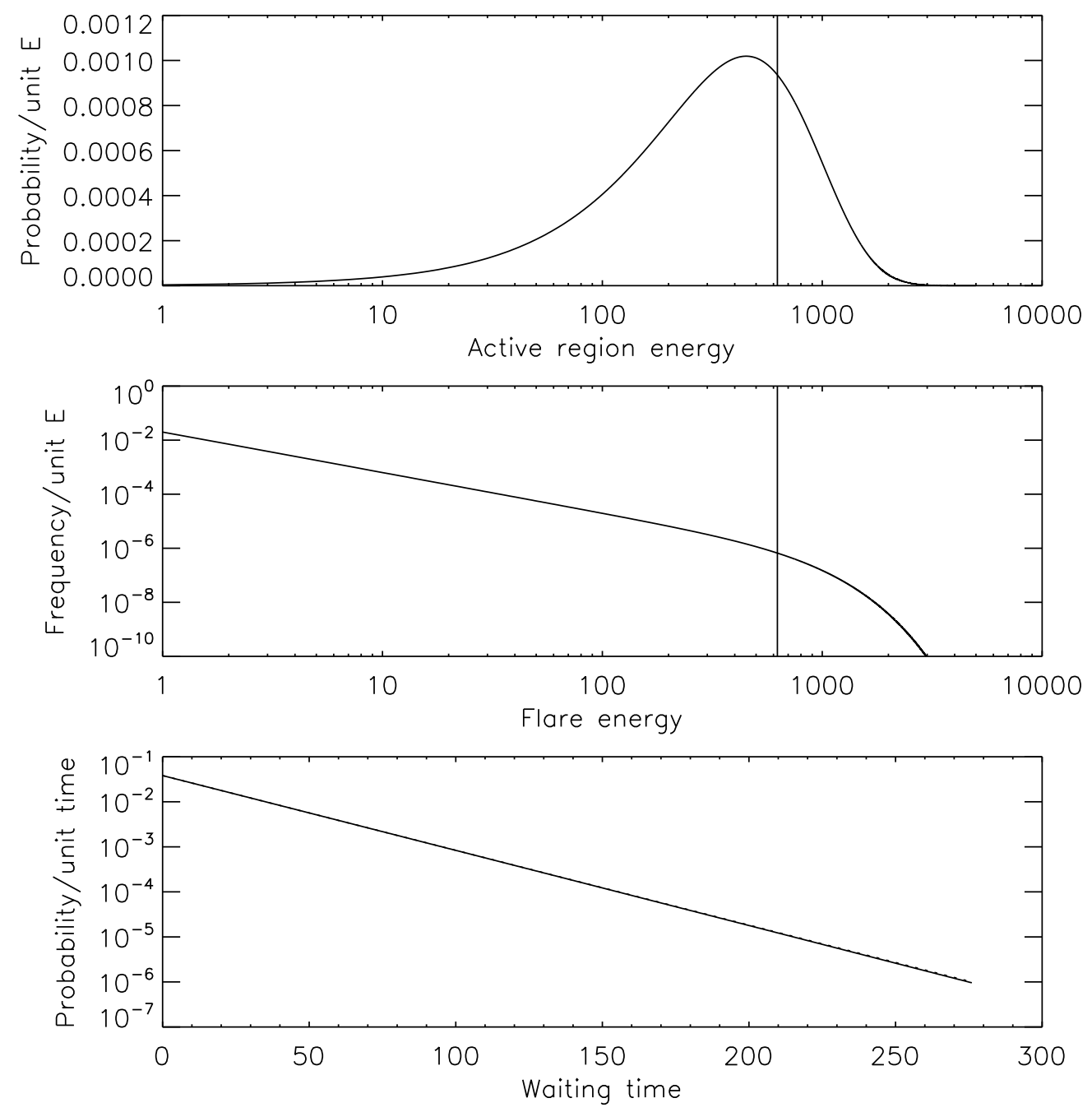

Fig. 2.- Numerical solution to the steady-state master equation for the case $\delta=0, \gamma=$ 1.5, and $\alpha_{0}=0.02$, one of the cases considered in Wheatland \& Glukhov (1998). Upper panel: probability distribution for free energy $P(E)$; middle panel: flare frequency-energy distribution $\mathcal{N}(E)$; lower panel: flare waiting-time distribution $p_{\tau}(\tau)$. 


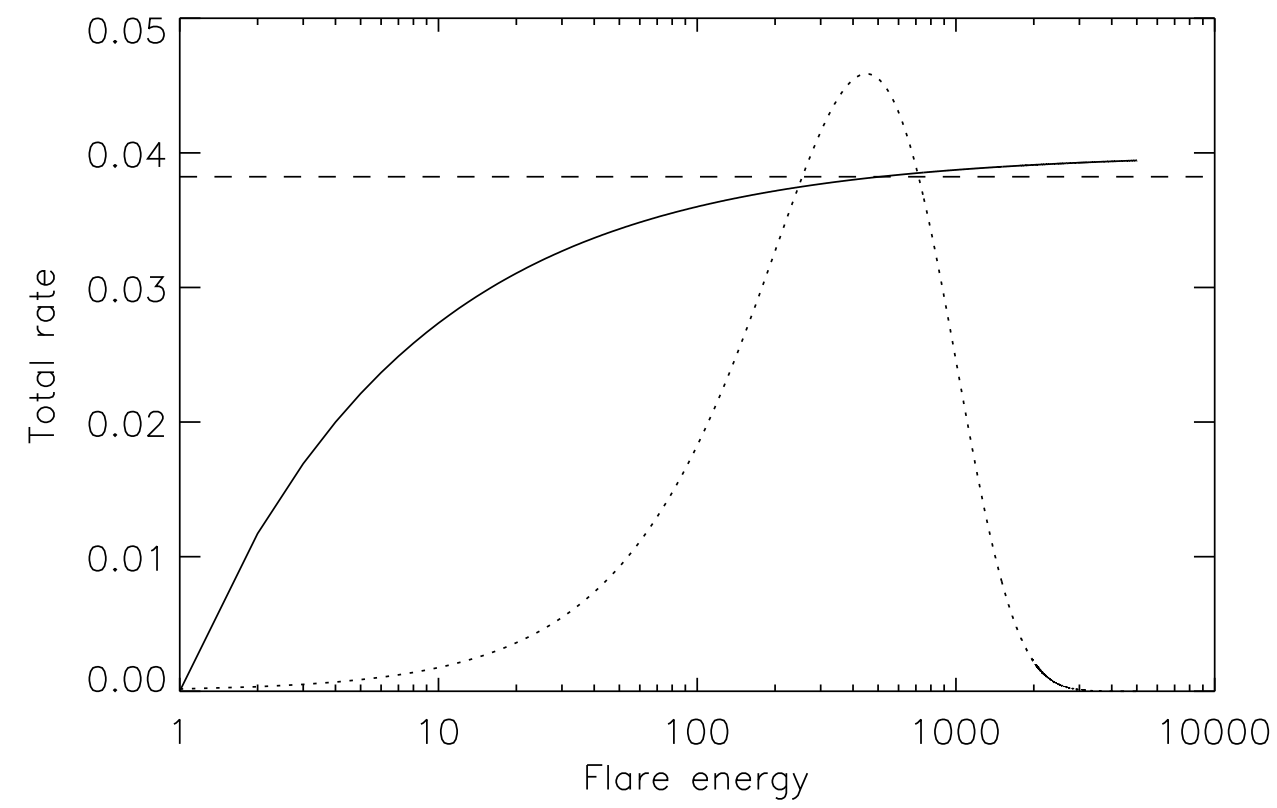

Fig. 3. - The total rate of flaring $\lambda(E)$ versus energy (solid curve) for the case $\delta=0$, $\gamma=1.5$, and $\alpha_{0}=0.02$, and the mean total rate $\langle\lambda\rangle$ (dashed line). The energy distribution $P(E)$ is also shown, with an arbitrary normalization (dotted curve). 

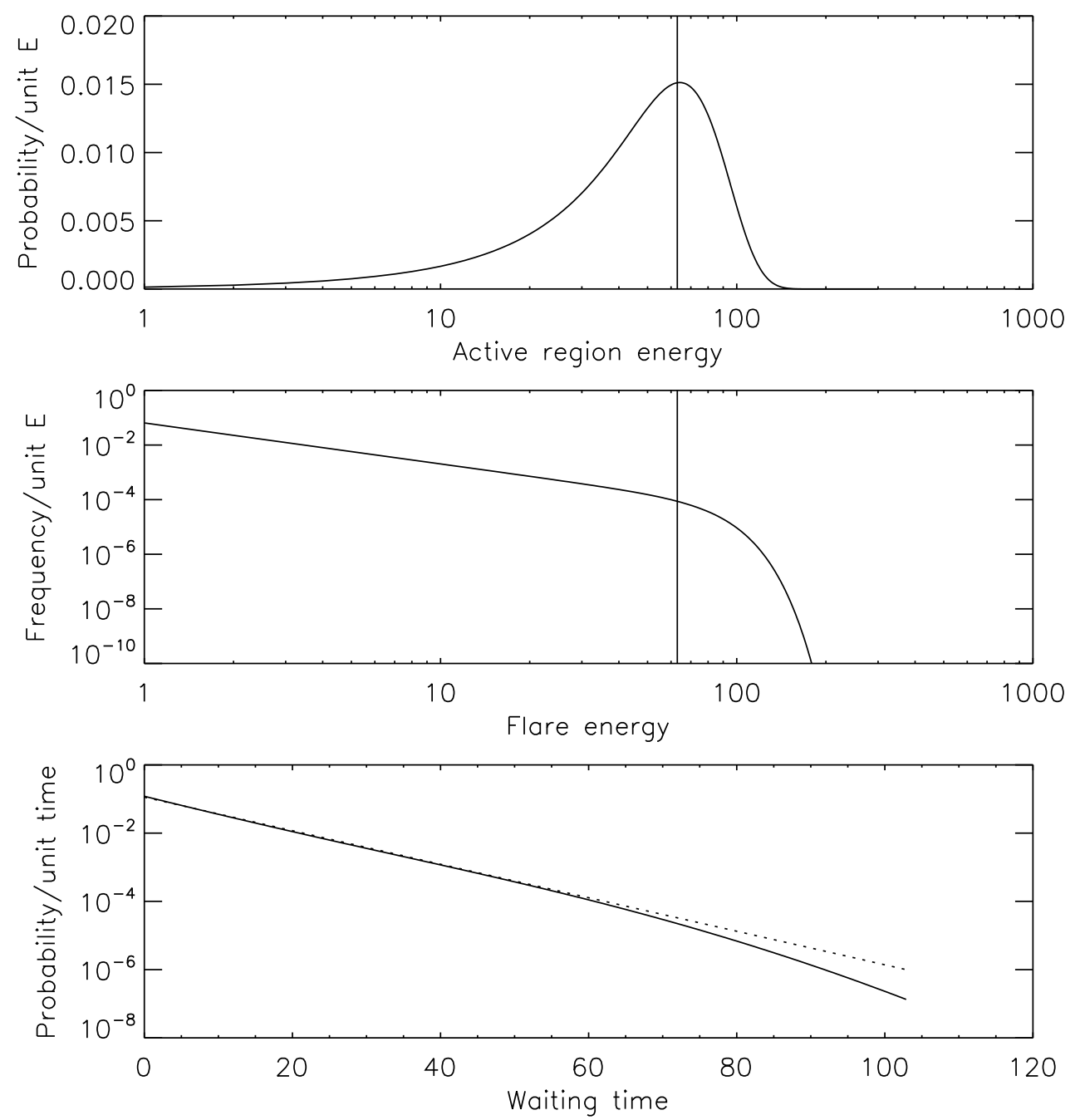

Fig. 4.- Numerical solution to the steady-state master equation for the case $\delta=1, \gamma=1.5$, and $\alpha_{0}=10^{-3}$. Upper panel: probability distribution for free energy $P(E)$; middle panel: flare frequency-energy distribution $\mathcal{N}(E)$; lower panel: flare waiting-time distribution $p_{\tau}(\tau)$. 

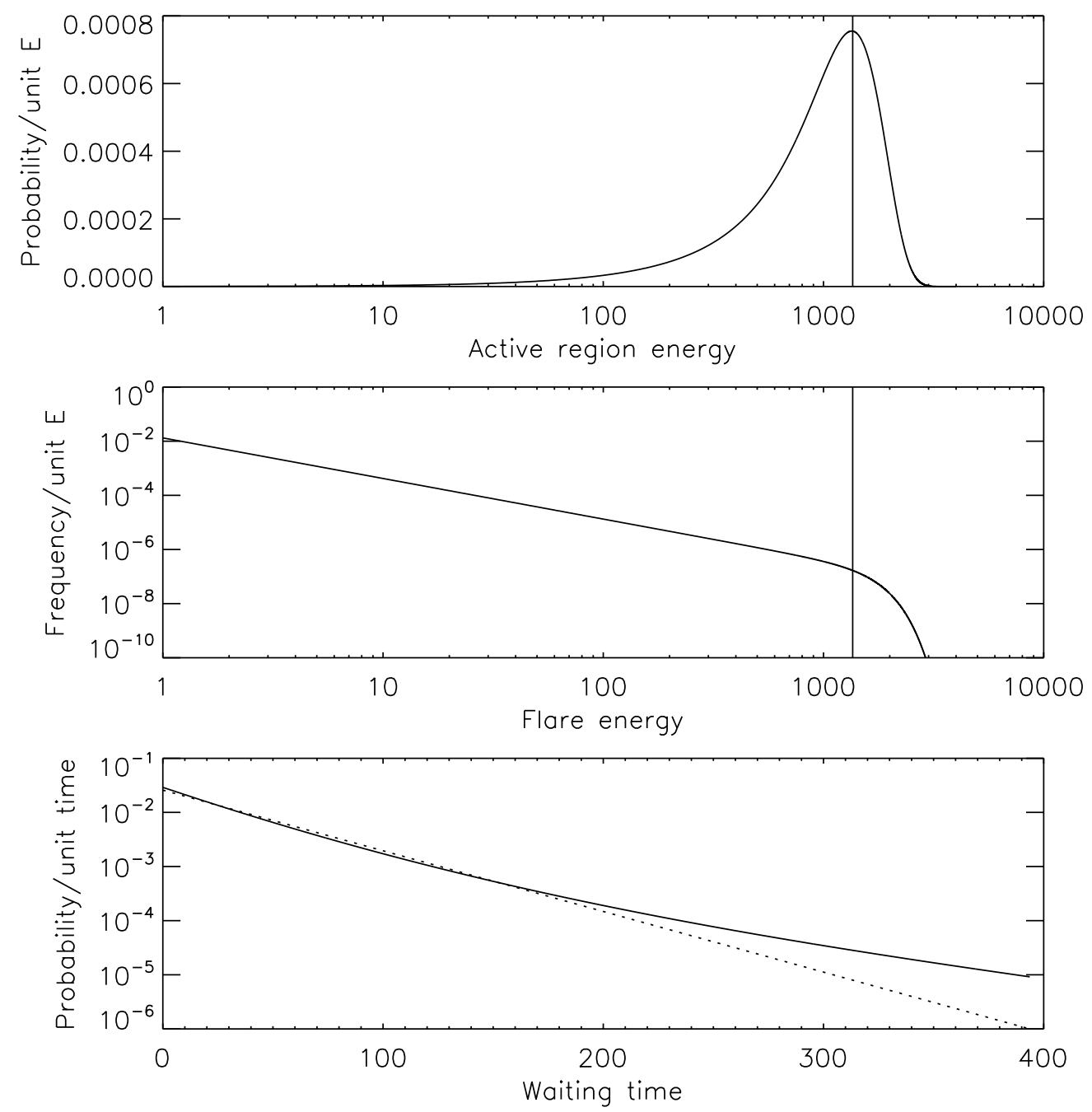

Fig. 5.- Numerical solution to the steady-state master equation for the case $\delta=1, \gamma=1.5$, and $\alpha_{0}=10^{-5}$. Upper panel: probability distribution for free energy $P(E)$; middle panel: flare frequency-energy distribution $\mathcal{N}(E)$; lower panel: flare waiting-time distribution $p_{\tau}(\tau)$. 


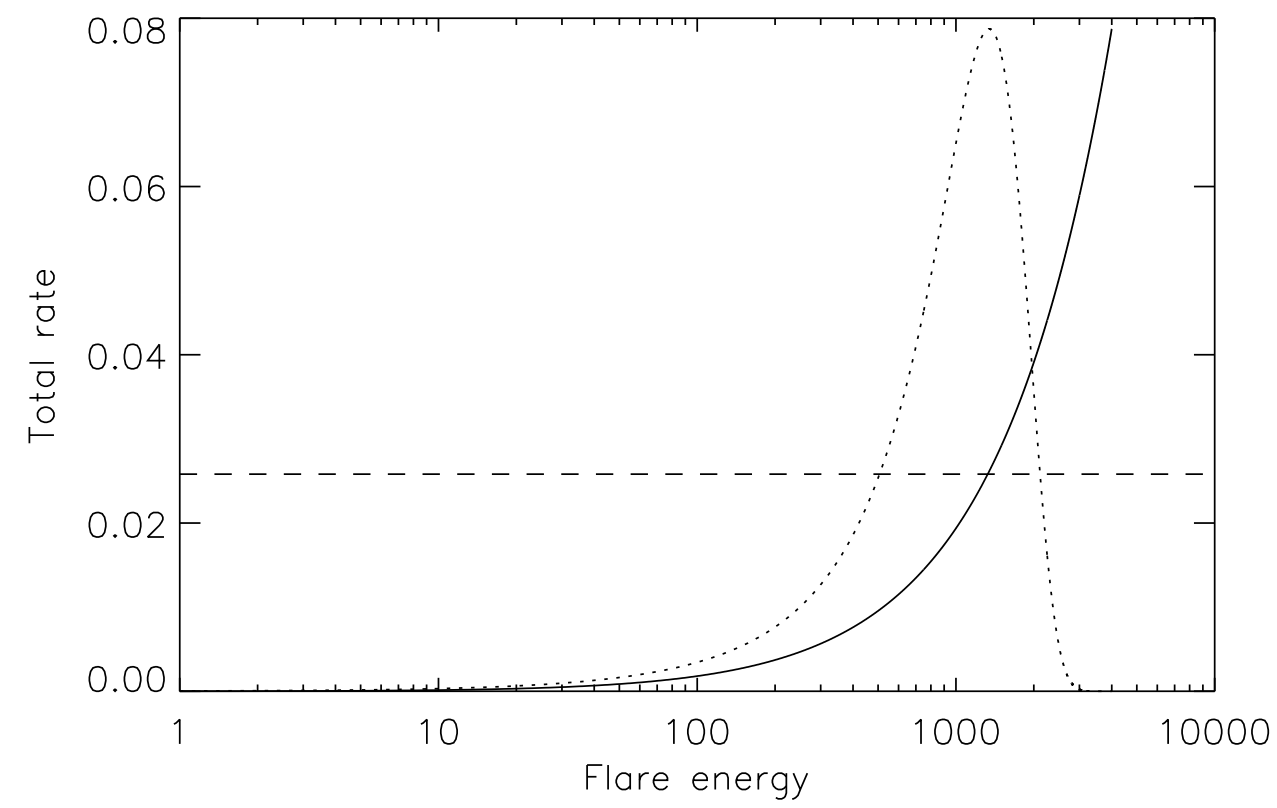

Fig. 6. - The total rate of flaring $\lambda(E)$ versus energy (solid curve) for the case $\delta=1$, $\gamma=1.5$, and $\alpha_{0}=0.02$, and the mean total rate $\langle\lambda\rangle$ (dashed line). The energy distribution $P(E)$ is also shown, with an arbitrary normalization (dotted curve). 